\title{
In vitro cytotoxic effects of secondary metabolites of DEHP and its alternative plasticizers DINCH and DINP on a L929 cell line
}

\section{Teuta Eljezi, ${ }^{a, b, 1}$ Pierre Pinta, ${ }^{c, 1}$ Fabien Nativel ${ }^{c}$ Damien Richard, ${ }^{d}$ Jérémy Pinguet, $^{d}$

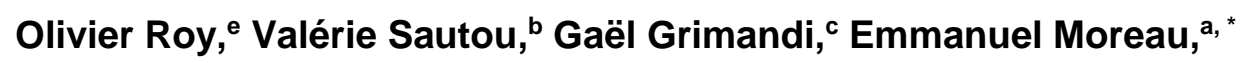

a Université Clermont- Auvergne, Imagerie Moléculaire et Stratégies Théranostiques, BP 184, F-63005 Clermont-Ferrand, France. Inserm, U 1240, F-63000 Clermont-Ferrand, France. Centre Jean Perrin, F-63011 Clermont-Ferrand, France.

b Université Clermont Auvergne, CHU Clermont Ferrand, CNRS, SIGMA Clermont, ICCF, F-63000 Clermont-Ferrand, France

c Inserm, UMR 1229, Regenerative Medicine and Skeleton (RMeS), Université de Nantes, ONIRIS, 44042 Nantes, France

d Service de Pharmacologie, CHU de Clermont-Ferrand, Clermont-Ferrand, France

e Université Clermont Auvergne, CNRS, SIGMA Clermont, ICCF, F-63000 Clermont-Ferrand, France. *corresponding author: emmanuel.moreau@uca.fr

${ }^{1}$ both authors contributed equally

\section{Abstract}

Background: Phthalic acid esters are widely used to improve the plasticity of PVC in medical devices (MD). The most famous plasticizer is DEHP, whose use in medical devices has been contested by the European authorities since 2008. Several alternative plasticizers are being considered to replace DEHP, such as DEHT, TOTM, DINP or DINCH, but they are also released from the PVC throughout their life cycle and are metabolized in the same way as DEHP.

Objectives: Our study focuses on the in vitro cytotoxicity of two alternative plasticizers (DINCH and DINP) contained in certain medical devices. They are likely to migrate and be transformed in vivo into the primary and secondary metabolites by a metabolism similar to that of DEHP. This preliminary study is the first to assess the in vitro cytotoxicity of oxidized metabolites of DINCH and DINP based on the EN ISO 10-993-5 standards documents.

Methods: We have studied the complete multi-step organic synthesis of secondary metabolites of DINP and DINCH and have performed cytotoxicity tests on L929 murine cells according to the ISO-EN ISO 10993-5 standard design for the biocompatibility of a MD. The tested concentrations of obtained metabolites $(0.01,0.05$ and $0.1 \mathrm{mg} / \mathrm{mL})$ covered the range likely to be found for DEHP (total metabolism) in biological fluids coming into direct contact 
with the MD. The concentrations tested in our study were chosen based on a complete transformation of the plasticizers released after direct contact between a MD and the patient's blood.

Results

Only 7-oxo-MMeOCH is cytotoxic at the highest concentration $(0.1 \mathrm{mg} / \mathrm{mL})$ after 7 days of exposure, just like 5-oxo-MEHP for the same concentration. By contrast, 7-OH-MMeOP, 7cx-MMeOP, 7-oxo-MMeOP, 7-OH-MMeOCH and 7-cx-MMeOCH were not found to be cytotoxic.

Conclusion: The known concentrations of these secondary metabolites in urinary samples are in the $\mu \mathrm{g} / \mathrm{L}$ range, i.e. about 100-1000 times lower than the concentrations tested in this study. Cytotoxicity is known to be dose-dependent but it is not always the case for endocrine perturbations and the secondary metabolites could induce endocrine perturbations at very low doses.

\section{Keywords}

DINCH, DINP, metabolite synthesis; cytotoxicity, DEHP-alternative plasticizers

\section{Abbreviations}

ARMED®, Assessment and Risk Management of Medical Devices in Plasticized Polyvinylchloride; DEHP, diethylhexyl phthalate; DINCH, diisononyl cyclohexane-1,2dicarboxylate; DINP, di-isononylphthalate; MEHP, monoethylhexyl phthalate; MMeOP, mono(4-methyloctyl)phthalate; $\quad \mathbf{M M e O C H}$, mono(4-methyloctyl) cyclohexane-1,2dicarboxylate; MINCH, monoisononyl cyclohexane-1,2-dicarboxylate; MINP, monoisononylphthalate; 7-oxo-MMeOCH, mono(4-methyl-7-oxooctyl) cyclohexane-1,2dicarboxylate, 7-OH-MMeOCH mono(7-hydroxy-4-methyl-octyl) cyclohexane-1,2dicarboxylate, 7-cx-MMeOCH, mono(7-carboxy-4-methyl-octyl) cyclohexane-1,2dicarboxylate, 7-oxo-MMeOP, mono(4-methyl-7-oxooctyl)phthalate, 7-OH-MMeOP mono(7hydroxy-4-methyloctyl)phthalate, 7-cx-MMeOP, mono(7-carboxy-4-methyloctyl)phthalate SCENIHR, Scientific Committee on Emerging and Newly-Identified Health Risks.

\section{INTRODUCTION}

Some phthalate esters present a general concern for public health due to their wide use in the manufacturing of polymeric materials and various consumer products. Some phthalate esters can be classified into two categories according to the number of carbons in the ester chain. On the one hand, there are those with a low molecular weight (number of carbon 
inferior to 6) which are used as industrial solvents, lubricants, and as components in personal-care products. On the other hand, those with a high molecular weight are commonly used as plasticizers, conferring better flexibility and durability to everyday polyvinyl chloride (PVC) consumer products, such as flooring, food packaging, clothing, children's toys and medical devices (Hauser and Calafat, 2005). These PVC plasticizers can be released from the polymers by volatilization into the air, abrasion of the polymer, leaching into liquids and direct diffusion from the polymer into dust on the polymer surface, resulting in subsequent human exposure which might cause adverse health effects (Giovanoulis $\mathrm{G}$ et al. 2018; Zhang, S. et al. 2016; Engel, A. et al., 2017, 2018). With regard to their presence in PVC medical devices, the most famous plasticizers is DEHP, whose use has been contested by the European authorities (SCENHIR, 2008, 2016). The toxic potential of DEHP is largely due to the metabolic transformation into more toxic metabolites by hydrolysis (MEHP) and subsequent oxidation reactions (5-OH-MEHP, 5-oxo-MEHP, 5-cx-MEHP) (Figure 1A). Numerous studies have been carried out on DEHP, leading to its classification as carcinogenic, mutagenic and or toxic for reproduction (CMR1B) because of its toxic effects on reproduction and fertility in rodents. Its use is now highly controlled in medical devices. The European regulation $2017 / 745$ recommends not to exceed a rate of $0.1 \%$ in mass fraction in each medical device (Regulation (EU) 2017/745). Two alternative plasticizers (diisononyl phthalate (DINP) and 1,2-cyclohexane dicarboxylic acid (DINCH)) have been considered by manufacturers as a replacement for DEHP in many PVC products, such as medical devices and food packaging, but not in toys are limited in toys with concentrations inferior to $0.1 \%$ by weight of the plasticized material (European Chemical Agency, 2013; SCENHIR, 2016), due to their physicochemical properties suggesting a lower migration and more favorable toxicological profile (Fromme et al 2016, Bhat et al 2014) than DEHP. DINP and DINCH respectively constitute a complex commercial mixture composed mainly of isomers of phthalate or dialkyl cyclohexanoate with 9 carbon chains. DINCH (Hexamoll@) is manufactured by catalytic hydrogenation of the aromatic ring of diisononyl phthalate (Palatinol® $\mathrm{N}$ ) into the cyclohexane ring existing predominantly in the most stable chair configuration, allowing cis- and trans-configuration. The typical commercial products consist of $90 \%$ cis- and $10 \%$ trans-isomers. In both molecules, the 4-methyl isomer forms a fraction that represents less than $50 \%$ of the total C9 alcohols (Schütze et al. 2017). Like DEHP, they are not covalently bonded to the PVC to which they are mixed. As a result, they are released from the products throughout their life cycle (Bernard et al 2015; Jeon et al. 2016). Because they are a mixture of isomers, their metabolites are also isomeric mixtures (Silva et al. 2013). Both are initially hydrolyzed in rodents and humans into their monoester (MINP or MINCH), followed by oxidative processes into several metabolites including mono-hydroxy, mono-oxo 
and mono-carboxymetabolites (Silva et al., 2007a, 2007b, Koch et al. 2007a, 2007b, 2013) (Figure 1B).

Some biomonitoring studies carried out in humans have made it possible to demonstrate the presence of DINCH and DINP metabolites in biological media such as urine, blood and nails (Gianouvoulis et al. 2016, 2018, Correia-Sa et al. 2017, Schütze et al. 2012, 2017, Zeman et al. 2013; Koch et al. 2017, Johns LE 2015). However, despite the animal toxicology data and the in vitro toxicity of the plasticizers, there is almost no information on the in-vitro toxicity of the oxidized metabolites of DINP and DINCH from medical devices except the urinary metabolites of DINCH, which seem to have an impact on the activities of the human nuclear receptors ERa, ER $\beta, A R$, PPARa and PPARy. However, the in vitro data does not support the notion that DINCH or any of the investigated metabolites may exert considerable endocrine effects in vivo at relevant human exposure levels. (Engel et al. 2017 and 2018). In a previous study we investigated the cytotoxicity of DEHP, DINCH, DINP and their corresponding primary unconjugated metabolites (MEHP, MINCH, MMeOCH, MINP, respectively), and showed that the primary metabolites are more cytotoxic than their corresponding plasticizer at concentrations between 0.01 and $0.1 \mathrm{mg} / \mathrm{mL}$. Only MMeOP, the primary metabolite of DINP, is not cytotoxic at these concentrations (Eljezi et al., 2017). Taking into account that DINP and DINCH are metabolized in the same way as DEHP, secondary metabolites are also found in the urine (7-oxo-MMeOCH, 7-OH-MMeOCH, 7-cx$\mathrm{MMeOCH}, 7-0 x 0-M M e O P, 7-O H-M M e O P$ and 7-cx-MmeOP) in a glucuroconjugated form or not. According to the data in the literature, it appears that $26 \%$ of $\mathrm{cx}-\mathrm{MINCH}$ are excreted in its unconjugated form in urine (Koch et al., 2013). These results are similar to the previous work concerning DEHP and DINP (Silva et al., 2003). In continuation of our work on alternative plasticizers to DEHP, we therefore undertook the total synthesis of the secondary metabolites of DINP and DINCH in order to be able to carry out a first cytotoxicity study on the NCTC clone 929 in accordance with the EN ISO 10-993-5 standards documents. 
<smiles>[R]OC(=O)c1ccccc1C(=O)O[R]</smiles>
DEHP

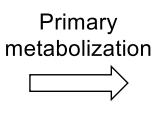

abolization

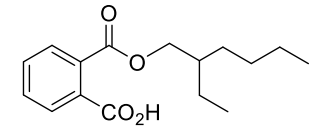

MEHP

B)

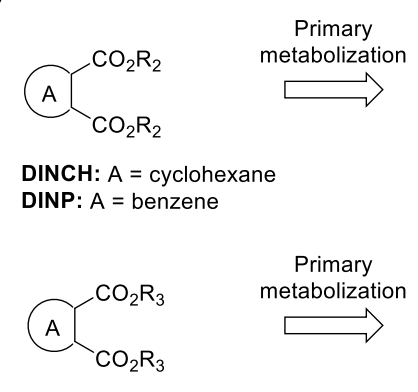

MeDINCH: $A=$ cyclohexane MeDINP: $A$ = benzene

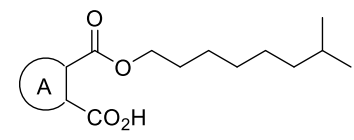

MINCH: A = cyclohexane MINP: $A=$ benzene

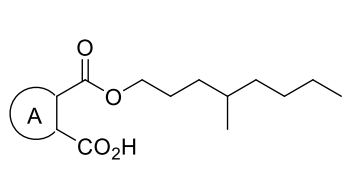

MMeOCH: $A$ = cyclohexane MMeOP: $A$ = benzene

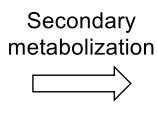

abolization

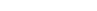

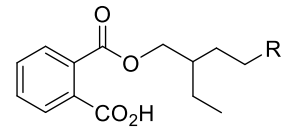

5-OH-MEHP: $\mathrm{R}=\mathrm{CH}(\mathrm{OH}) \mathrm{CH}_{3}$

5-oxo-MEHP: $\mathrm{R}=\mathrm{C}(\mathrm{O}) \mathrm{CH}_{3}$

5-cx-MEHP: $\mathrm{R}=\mathrm{CH}_{2} \mathrm{CO}_{2} \mathrm{H}$

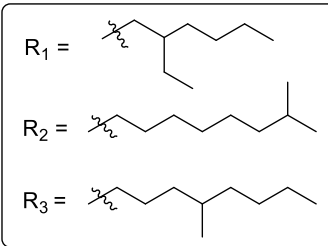

Secondary metabolization<smiles>[R]CCC(C)CCCOC(=O)c1ccccc1C(=O)O</smiles>

5-OH-MMeOCH: $\mathrm{A}=$ cyclohexane; $\mathrm{R}=\mathrm{CH}(\mathrm{OH}) \mathrm{CH}_{3}$ 5-OH-MMeOP: $A$ = benzene; $\mathrm{R}=\mathrm{CH}(\mathrm{OH}) \mathrm{CH}_{3}$

5-oxo-MMeOCH: $\mathrm{A}=$ cyclohexane; $\mathrm{R}=\mathrm{C}(\mathrm{O}) \mathrm{CH}_{3}$ 5-oxo-MMeOP: $\mathrm{A}$ = benzene; $\mathrm{R}=\mathrm{C}(\mathrm{O}) \mathrm{CH}_{3}$

5-cx-MMeOCH: $\mathrm{A}=$ cyclohexane; $\mathrm{R}=\mathrm{CH}_{2} \mathrm{CO}_{2} \mathrm{H}$ 5-cx-MMeOP: $A$ = benzene; $\mathrm{R}=\mathrm{CH}_{2} \mathrm{CO}_{2} \mathrm{H}$

Figure 1: Primary and secondary metabolites of DEHP, DINCH, DINP, MeDINCH and MeDINP

\section{MATERIALS AND METHODS}

\subsection{Chemicals, biochemicals and reagents}

Unless otherwise mentioned, all manipulations were performed under an argon atmosphere. All reagents were purchased from the following commercial suppliers: Sigma-Aldrich, Acros Organics, Carlo Erba, TCl Europa, and Alpha Aesar. Anhydrous DMF and anhydrous triethylamine were purchased from Acros Organics. THF was distilled over benzophenone and sodium. Dichloromethane was distilled over calcium hydride. Nuclear magnetic resonance (NMR) spectra were acquired on a Bruker AC-200 or AC-400 instrument operating at $200 \mathrm{MHz}$ and $50 \mathrm{MHz}$ or $400 \mathrm{MHz}$ and $100 \mathrm{MHz}$ for $1 \mathrm{H} \mathrm{NMR}$ and 13C NMR, respectively. All $1 \mathrm{H}$ NMR and $13 \mathrm{C}$ NMR spectral peaks are reported in $\delta$ units, parts per million (ppm), and the coupling constants are indicated in hertz $(\mathrm{Hz})$. The following abbreviations are used for spin multiplicity: $\mathrm{s}=$ singlet, $\mathrm{d}=$ doublet, $\mathrm{t}=$ triplet, $\mathrm{q}=$ quadruplet, $\mathrm{m}=$ multiplet, and $\mathrm{br}=$ broad. The chromatographic system consisted of an HTC PAL autosampler, and a Transcend TLX-1 HTLC System (ThermoFisher Scientific, San Jose, United States). The HRMS analysis was performed using a Thermo Exactive benchtop Orbitrap ${ }^{\circledR}$ instrument. TLC was performed on pre-coated silica gel sheets (POLYGRAM® 60F254 plates) and visualized under UV light (254 nm). Revelators used were $\mathrm{KMnO} 4$ (1.5 g $\mathrm{KMnO}_{4}, 10 \mathrm{~g} \mathrm{~K}_{2} \mathrm{CO}_{3}$, and $1.25 \mathrm{~mL} 10 \% \mathrm{NaOH}$ in $200 \mathrm{~mL}$ water) and ninhydrin (1.5 g 
ninhydrin in $100 \mathrm{~mL}$ of $\mathrm{n}$-butanol with $3 \mathrm{~mL} \mathrm{AcOH}$ ). Column chromatography was performed using a silica gel normal phase $(35-70 \mu \mathrm{m})$. A HPLC Prominence-i LC-2030 CSCHIMADZU with Kinetex $5 \mu \mathrm{m}$ EVO C18 (Phenomenex) column with pore diameter (A) 82-102, particle size $(\mu \mathrm{m}) 4.5-4.8$, and column dimensions of $50 \times 4.6 \mathrm{~mm}$ was used to purify secondary metabolites. Uncorrected melting points (Mp) were recorded on an Electrothermal IA9300 apparatus. Infrared spectra (IR) were recorded on a Bruker FT Vector 22. The 5-oxo-MEHP, 5-OH-MEHP and 5-cx-MEHP compounds were synthesized using the procedure described by Nüti et al. (Nüti et al, 2005).

Dimethylsulfoxide DMSO (ref: D8418 CAS: 67-68-5), Mouse fibroblasts L929 (ref: 85011425 lot: 10L019), MTT assay (3-(4,5-dimethylthiazol-2-yl)-2,5-diphenyl tetrazolium bromide, ref: M2128) were also purchased from Sigma Aldrich. Dulbecco's modified Eagle Medium (DMEM) (fetal calf serum (SVF), phosphate saline buffer (PBS), penicillin (P), streptomycin $(S)$, and actinomycin D) were purchased from Life technologies SAS, France. For more details, see supporting information.

\subsection{Methods for the synthesis of the secondary metabolites 7-oxo-MMeOCH, 7-OH- MMeOCH, 7-cx-MMeOCH, 7-oxo-MMeOP, 7-OH-MMeOP and 7-cx-MMeOP}

Metabolites 7-oxo-MMeOCH, 7-OH-MMeOCH, 7-cx-MMeOCH, 7-oxo-MMeOP, 7-OHMMeOP and 7-cx-MMeOP were synthesized from the appropriate anhydrides (hexahydroisobenzofuran-1,3-dione or phtalic anhydride) and 7-(benzyloxy)-4-methylheptan1-ol previously synthesized and characterized $\left({ }^{1} \mathrm{H},{ }^{13} \mathrm{C}\right.$ NMR and HRMS) by our lab (Eljezi et al. 2017). The benzyl group at $\omega$-position was then removed under hydrogen atmosphere and the resulting alcohol was oxidized into an aldehyde compound which was converted into vinyl compounds 6 or 18. From the appropriate vinyl compound (6 or 18), 7-oxo-MMeOCH or 7-oxo-MMeOP could be obtained by oxidation in a mixture of $\mathrm{PdCl}_{2}$ and parabenzoquinone. These were directly converted into $7-\mathrm{OH}-\mathrm{MMeOCH}$ or $7-\mathrm{OH}-\mathrm{MMeOP}$ by a regioselective reduction in the presence of $\mathrm{NaBH}_{4}$. Concerning 7-cx-MMeOCH or 7-cx-MMeOP, they were obtained from vinyl derivatives 6 or $\mathbf{1 8}$ by hydroboration followed by an oxidation in the presence of Jones reagent. The purity of all synthesized metabolites was over 95\% (HPLC/MS). All experimental details are described in the supporting material. 


\subsection{Biocompatibility assays}

For the solubilization of the plasticizers, cell cultures, viability assays for the synthesized metabolites and statistical analysis, we used the same procedures previously described by us (Eljezi et al. 2017). For more details, see supporting information.

\section{RESULTS}

\section{Synthesis of MeDINCH and MeDINP secondary metabolites}

The three metabolites of the MeDINCH plasticizer, i.e. 7-oxo-MMeOCH, 7-OH-MMeOCH and 7-cx-MMeOCH, were synthesized from monoprotected alcohol 1 (Eljezi et al.2017) and hexahydroisobenzofuran-1,3-dione (Scheme 1). Acid compound 2 was esterified, followed by a catalytic hydrogenation to produce compound $\mathbf{4}$. The hydroxy group at $\omega$-position of the alkyl chain was oxidized into an aldehyde. Compound $\mathbf{5}$ reacted with isopropyltriphenylphosphonium iodide (Wittig reaction) to obtain vinyl derivative $\mathbf{6}$. According to the procedure previously described (Nüti et al. 2005), this vinyl function produced a ketone function at position C-7 of the alkyl chain (compound 7) which was converted into 7-oxo$\mathrm{MMeOCH}$ after a soft saponification. Compound 7 was also reduced in the presence of $\mathrm{NaBH}_{4}$ followed by a treatment with lithium hydroxide monohydrate to give 7-OH-MMeOCH. Finally, the third secondary metabolite, 7-cx-MMeOCH, was obtained from compound $\mathbf{6}$ after a hydroboration of the vinyl function, followed by an oxidation of the primary alcohol function with Jones reagent and a soft saponification. All of these syntheses are depicted in Scheme 1.

Scheme 1. Synthesis of secondary metabolites 7-oxo-MMeOCH, 7-OH-MMeOCH and 7cX-MMeOCH 


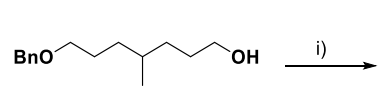

(1)

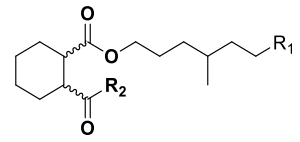

ii) (2): $\mathrm{R}_{1}=\mathrm{CH}_{2} \mathrm{OBn}, \mathrm{R}_{2}=\mathrm{OH}$ (3): $\mathrm{R}_{1}=\mathrm{CH}_{2} \mathrm{OBn}, \mathrm{R}_{2}=\mathrm{OEt}$ iii) (4): $\mathrm{R}_{1}=\mathrm{CH}_{2} \mathrm{OH}, \mathrm{R}_{2}=\mathrm{OEt}$ iv) $C_{(5): R_{1}}=C H O, R_{2}=O E t$<smiles>CC(=O)CCC(C)CCCOC(=O)[C@@H]1CCCC[C@H]1C(=O)O</smiles>

(8)

7-oxo-MeMINCH

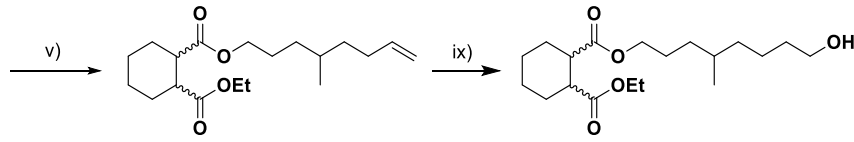

(6)

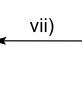<smiles>CCOC(=O)C1CCCCC1C(=O)OCCCC(C)CCC(C)=O</smiles>

(7)<smiles>CCOC(=O)C1CCCCC1C(=O)OCCCC(C)CCC(C)O</smiles>

0
(9)

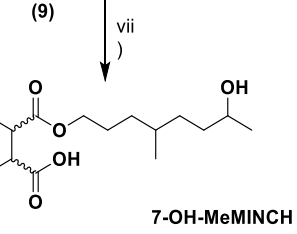

(10) viii

vi

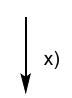<smiles>CCOC(=O)C1CCCCC1C(=O)OCCCC(C)CCCC(=O)O</smiles>

(12) vii)

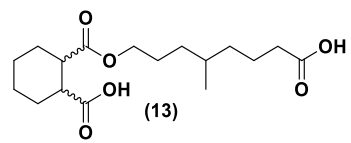

7-cx-MeMINCH

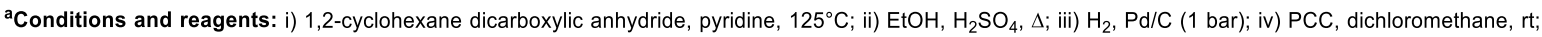
v) isopropyltriphenylphosphonium iodide, $\mathrm{n}-\mathrm{BuLi}, \mathrm{THF},-78^{\circ} \mathrm{C}$ to rt; vi) $\mathrm{PdCl} 2, \mathrm{p}$-benzoquinone, $\mathrm{DMF} / \mathrm{H}_{2} \mathrm{O}(7 / 1 ; \mathrm{v} / \mathrm{v})$, rt; vii) $\mathrm{LiOH}(1 \mathrm{M}), \mathrm{THF} / \mathrm{methanol}(3 / 1 ; \mathrm{v} / \mathrm{v})$, rt; viii) $\mathrm{NaBH}_{4}$, ethanol, rt; ix) $\mathrm{B}_{2} \mathrm{H}_{6}(1 \mathrm{M})$, THF, rt; $\mathrm{x}$ ) Jones reagent, rt;

The secondary metabolites 7-oxo-MMeOP, 7-OH-MMeOP and 7-cx-MMeOP were synthesized using a similar strategy developed for secondary metabolites of MeDINCH (Scheme 2). Briefly, vinylic precursor 18 was obtained by condensation of the monoprotected alcohol 1, and anhydride phthalic, followed by an esterification, deprotection of the $\omega$-hydroxy group at C-7 position, then its oxidation and a Wittig reaction. Finally, this vinyl function enabled 7-oxo-MMeOP to be obtained in two steps (Wacker oxidation and a soft saponification), then 7-OH-MMeOP by a reduction in presence of $\mathrm{NaBH}_{4}$. Compound 7-cxMMeOP was obtained after a hydroboration, followed by an oxidation with Jones reagent.

Scheme 2. Synthesis of secondary metabolites 7-oxo-MMeOP, 7-OH-MMeOP and 7-cxMMeOP. 


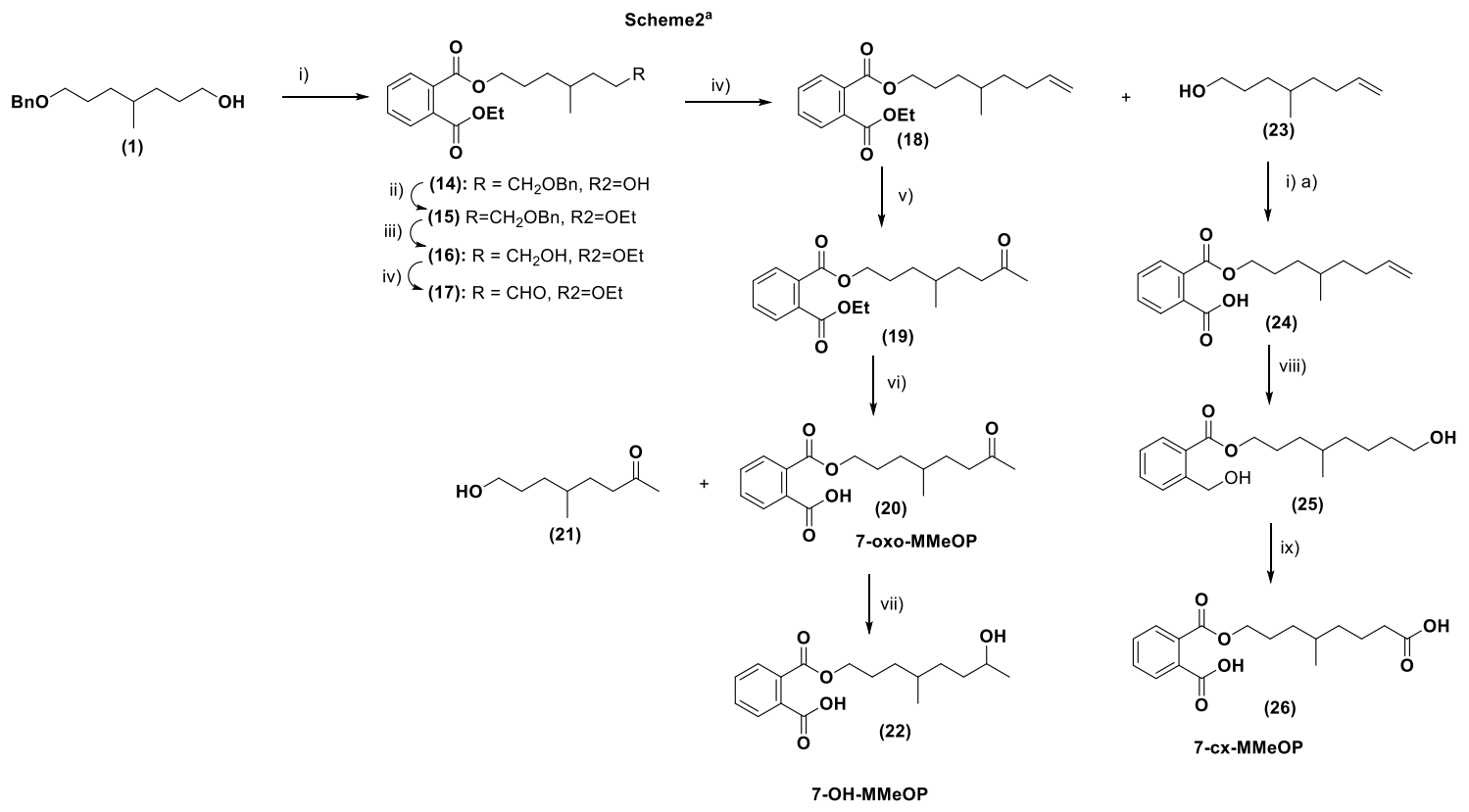

aConditions and reagents: i) a) phthalic anhydride, pyridine, $125^{\circ} \mathrm{C}$; b) $\mathrm{EtOH}, \mathrm{H}_{2} \mathrm{SO}_{4}, \Delta$; ii) $\mathrm{H}_{2}, \mathrm{Pd} / \mathrm{C}$ (1 bar); iii) $\mathrm{PCC}$, dichloromethane, rt iv) isopropyltriphenylphosphonium iodide, $n-B u L i, T H F,-78^{\circ} \mathrm{C}$ to $\mathrm{rt}$; v) $\mathrm{PdCl}$, p-benzoquinone, $\mathrm{DMF} / \mathrm{H}_{2} \mathrm{O}(7 / 1 ; \mathrm{v} / \mathrm{v})$, rt; vi) $\mathrm{LiOH}(1 \mathrm{M}), \mathrm{THF} / \mathrm{methanol}(3 / 1 ; \mathrm{v} / \mathrm{v}), \mathrm{rt}$ vii) $\mathrm{NaBH}_{4}$, ethanol, rt; viii) $B_{2} \mathrm{H}_{6}(1 \mathrm{M}), \mathrm{THF}, \mathrm{rt}$; ix) Jones reagent, rt:

3.2. Viability of cells when exposed to the secondary metabolites of plasticizers

Figure 2 shows the results of viability tests, at day 7 (D7), performed on L929 cells cultured in the presence of the three concentrations of plasticizers after 7 days of contact. Compounds 5-cx-MEHP, 5-OH-MEHP, 7-cx-MMeOCH, 7-OH- MMeOCH, 7-cx-MMeOP, 7OH-MMeOP, 7-oxo-MMeOP were not toxic to the cells at concentrations of $0.01,0.05$, and $0.1 \mathrm{mg} / \mathrm{mL}$ compared with the control containing DMSO at the same concentrations. A decrease in cell viability was observed for 5-oxo-MEHP and 7-oxo-MMeOCH from the concentration of $0.1 \mathrm{mg} / \mathrm{mL}$. A significant decrease in viability of over $50 \%$ was observed for 5-OXO-MEHP 
A

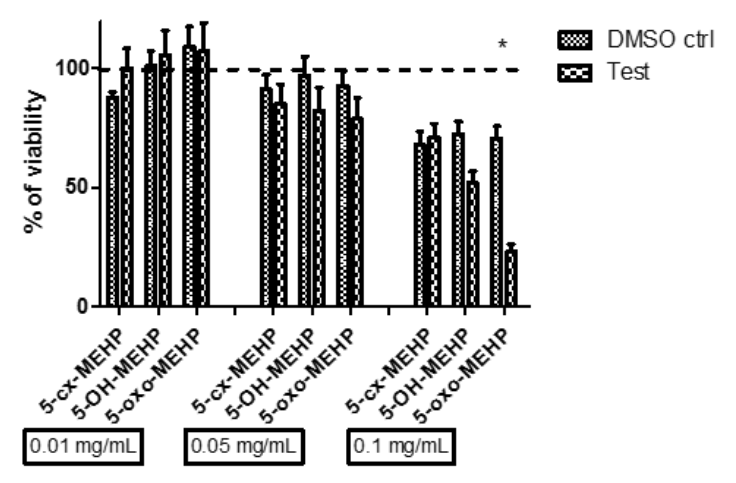

B

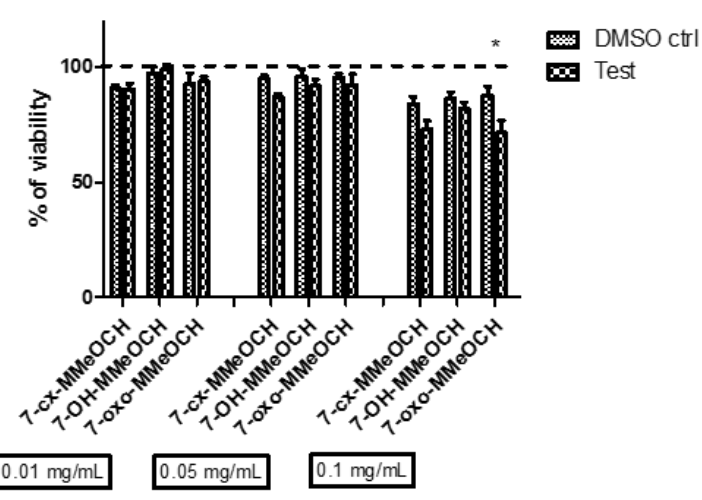

C

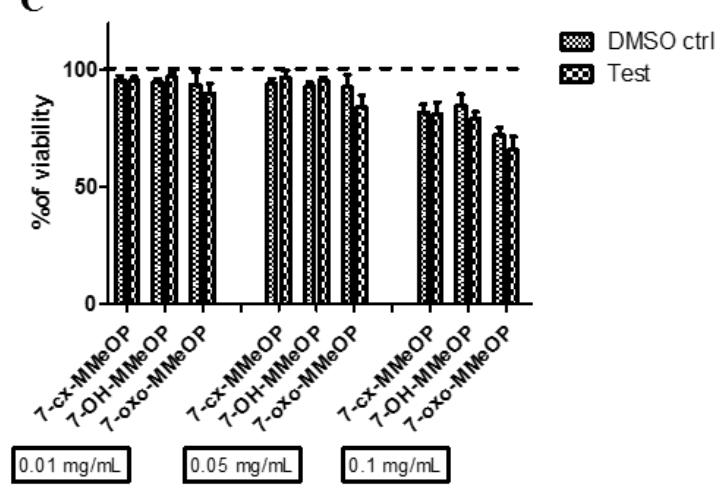

Figure 2: Cytotoxicity tests of secondary metabolites at $0.01 \mathrm{mg} / \mathrm{mL}, 0.05 \mathrm{mg} / \mathrm{mL}$ and $0.1 \mathrm{mg} / \mathrm{mL}$, on day 7. Outcomes of the cell viability analysis using a MTT assay. L929 cells were seeded at $10^{4}$ cells/well and cultured for 7 days in three conditions: control (complete medium), control DMSO (complete medium supplemented with $0.1 \%, 0.5 \%$ and $1 \% \mathrm{DMSO}$ ) and test (working solutions of the plasticizer in complete medium at $0.01 \mathrm{mg} / \mathrm{mL}, 0.05 \mathrm{mg} / \mathrm{mL}$ and $0.1 \mathrm{mg} / \mathrm{mL}$ ). MTT assays and quantification of the viability percentage were carried out every day. ${ }^{*} p<0.05$ DMSO control vs Test.

\section{DISCUSSION}


For several years, our research program has focused on DEHP and some of the corresponding alternatives in medical devices. DINCH and DINP are two plasticizers commonly used as alternatives to DEHP in PVC medical devices. DINCH is used in parenteral and enteral nutrition tubings as well as in hemodialysis lines (Strommen et al. 2016). DINP is added to some PVC infusions tubings or blood transfusion sets (Fredericks et al. 2014; Bernard et al. 2015; Bourdeaux et al. 2016). Like DEHP, these plasticizers are also known to migrate from medical devices and to enter into contact with the patient's body where they are metabolized into primary and secondary forms (Bernard et al. 2015). Based on data obtained from ICU studies carried out in recent years (Fredericksen Frederiksen et al. 2014; Huygh et al. 2015; Strommen et al. 2016), it seems that these metabolites are less present in the urine than those of DEHP. Although these metabolites are mainly eliminated in their conjugated form in the urine, about $26 \%$ of the acid form is in its free form ( $5 \mathrm{cx}-\mathrm{MINCH})$ (Koch et al., 2013). Similar data have been published for DEHP and DINP (Silva et al., 2003). The cytotoxicity of their respective primary metabolites (MINCH, MeMINCH, MINP and MeMINP) has also been evaluated (Eljezi et al. 2017) but the cytotoxicity of the secondary metabolites of DINCH and DINP have not currently been studied. We thus synthesized these secondary metabolites (7-oxo-MMeOCH, 7-OH-MMeOCH, 7-cxMMeOCH, 7-oxo-MMeOP, 7-OH-MMeOP and 7-cx-MMeOP) in sufficient quantities to complete preliminary cytotoxicity test. Based on the urinary concentrations found for these secondary metabolites (conjugates or not), the concentrations of the secondary metabolites of DEHP are much higher than those of DINP and DINCH $(0.3-5 \mu \mathrm{g} / \mathrm{mL}$ for $\Sigma$ DEHPm versus 0.008-0.1 $\mu \mathrm{g} / \mathrm{mL}$ for $\Sigma$ DINPm and $\Sigma$ DINCHm) (Frederiksen et al., 2014; Huygh et al., 2015; Strommen et al., 2016). We therefore evaluated the cytotoxicity of the secondary metabolites of DINP and DINCH over a larger concentration range $(0.01-1 \mathrm{mg} / \mathrm{mL})$, which is a very pessimistic scenario, and compared these results with those obtained for the secondary metabolites of DEHP. We chose this concentration range in order to take into account the fact that DINCH and DINP could be increasingly used in the composition of medical devices, thus exposure to them is expected to increase in the coming years. It must also be taken into consideration that patients in intensive care units are exposed to several medical devices that may contain these alternative plasticizers (Huygh et al. 2015; Strommen et al. 2016). Metabolites 7-OH-MMeOP, 7-OH-MMeOCH, 7-cx-MMeOP, 7-cx-MMeOCH do not cause any cytotoxicity up to concentrations of $0.1 \mathrm{mg} / \mathrm{mL}$, just like the corresponding secondary metabolites of DEHP, i.e. 7-OH-MEHP and 5-cx-MEHP. This is also true for 7-oxo-MMeOP, the third secondary metabolite of DINP. However, 7-oxo-MMeOCH, like 5-oxo-MEHP, has been shown to be cytotoxic at $0.1 \mathrm{mg} / \mathrm{mL}$. No cytototoxicity was observed at a concentration below $0.05 \mathrm{mg} / \mathrm{mL}$. The concentrations tested in our study were chosen on the basis of a complete transformation of the plasticizers released after direct contact between a MD and 
the patient's blood (SCENHIR 2015-2016, Eckert E et al., 2015, 2016). However, the secondary metabolites, derived from the hepatic metabolism of the primary metabolite of the plasticizer (itself derived from an enzymatic hydrolysis of the plasticizer) are present at much lower concentrations in body fluids. Human biomonitoring studies (Schütze et al. 2014, Koch et al. 2007a, 2007b) performed after the introduction of DINCH and DINP on to the market show that the concentrations of their secondary metabolites in urinary samples are in the $\mu \mathrm{g} / \mathrm{L}$ range (example $0.71 \mu \mathrm{g} / \mathrm{L}$ for 7-OH-MMeOCH, $0.33 \mu \mathrm{g} / \mathrm{L}$ for 7-oxo-MMeOCH, $0.61 \mu \mathrm{g} / \mathrm{L}$ for 7-cx-MMeOCH, $14.9 \mu \mathrm{g} / \mathrm{L}$ for 7-OH -MMeOP, $8.9 \mu \mathrm{g} / \mathrm{L}$ for 7-oxo-MMeOP and $16.4 \mu \mathrm{g} / \mathrm{L}$ for 7-cx-MMeOP), which is about 100-1000 times lower than the concentrations tested. The DEHP secondary metabolites found in the urine of humans exposed for two decades are also in the order of $\mu \mathrm{g} / \mathrm{L}(21.0 \mu \mathrm{g} / \mathrm{L}$ for 5-OH-MEHP, $16.7 \mu \mathrm{g} / \mathrm{L}$ for 5-oxo-MEHP, $26,9 \mu \mathrm{g} / \mathrm{L}$ for 5-cx-MEHP) (Wittassek et al. 2007). Therefore, we can consider that these secondary metabolites of DINCH and DINP do not present a risk of cytotoxicity at the doses found in the body. This information, combined with the toxicity data obtained in animals, is reassuring with regard to the use of MDs plasticized with DINCH or DINP for situations where there is no direct contact with the patient's biological fluids (infusers, extension lines, nutrition tubings). In the case of a direct contact between the plasticized PVC in medical device and the patient's blood (extracorporeal circulation in cardiac surgery, dialysis), the risk could be different. In our previous study, we showed the cytotoxicity of primary metabolites of DINP and DINCH at $0.05 \mathrm{mg} / \mathrm{mL}$ (Eljezi et al. 2017). During an extracorporeal circulation, the plasticizer released from the MD (extracorporeal circulation line) is hydrolyzed into its glucuroconjugated primary metabolite. However, studies on plasticized DINCH blood bags have shown that this does not affect plasma quality and the amount of DINCH released is less pronounced than that of DEHP (Lagerberg et al. 2015). These results seem to be in agreement with those obtained in rats after i.v. injection or infusion of DINCH for which no apparent systemic effect had been observed up to $300 \mathrm{mg} / \mathrm{kg}$ bw/day (David et al. 2015).

It should be noted that these preliminary results attempt to show that the secondary metabolites of DINCH and DINP do not exhibit cytotoxicity according to EN ISO 10-993-5 standards documents. We will consider these different aspects when our biomonitoring study on alternative plasticizers and their corresponding metabolites are complete within the framework of ARMED® and ARMED-NEO projects. We will then select the different compounds for complementary studies.

\section{Declaration of interest}

This study is a part of the ARMED® project and received financial support from the French National Agency for the Safety of Medicines and Health Products (ANSM). 


\section{References}

Bhat, V. S., Durham, J. L., Ball, G. L., English, J. C. 2014. Derivation of an oral reference dose $(\mathrm{RfD})$ for the nonphthalate alternative plasticizer 1,2-cyclohexane dicarboxylic acid, diisononyl ester (DINCH). J. Toxicol. Environ. Health. B. Crit. Rev. 17, 2, 63-94.

Bernard, L., Cueff, R., Chagnon, M., Abdoulouhab, F., Décaudin, B., Breysse, C., Kauffmann, S., Cosserant, B., Souweine, B., Sautou, V. 2015. Migration of plasticizers from PVC medical devices: Development of an infusion model. Int. J. Pharm. 494, 1, 136-145.

Bourdeaux, D., Yessaad, M., Chennell, P., Larbre, V., Eljezi, T., Bernard, L., Sautou, V. 2016. Analysis of PVC plasticizers in medical devices and infused solutions by GC-MS. J. Pharm. Biomed Anal. 118, 206-213.

Campioli E, Lee S, Lau M, Marques L, Papadopoulos V. 2017. Effect of prenatal DINCH plasticizer exposure on rat offspring testicular function and metabolism. Sci. Rep. 7, 1, 11072.

Correia-Sá, L., Schütze, A., Norberto, S., Calhau, C., Domingues, V. F., Koch, H. M. Exposure of Portuguese children to the novel non-phthalate plasticizer di-(iso-nonyl)cyclohexane-1,2-dicarboxylate (DINCH). 2017. Environ. Int. 102, 79-86.

David, R. M., White, R. D., Larson, M. J., Herman, J. K., Otter, R. 2015. Toxicity of HexamolI(®) DINCH(®) following intravenous administration. Toxicol Lett. 238, 2, 100-109.

Eckert, E., Müller, J., Göen, T. 2015. Simultaneous determination of polyvinylchloride plasticizers di(2-ethylhexyl) phthalate and tri(2-ethylhexyl) trimellitate and its degradation products in blood by liquid chromatography-tandem mass spectrometry. J. Chromatogr. A. 1410, 173-180.

Eckert E, Münch F, Göen T, Purbojo A, Müller J, Cesnjevar R. 2016. Comparative study on the migration of di-2-ethylhexyl phthalate (DEHP) and tri-2-ethylhexyl trimellitate (TOTM) into blood from PVC tubing material of a heart-lung machine. Chemosphere 145, 10-16.

Eljezi, T., Pinta, P., Richard, D., Pinguet, J., Chezal, J. M., Chagnon, M. C., Sautou, V., Grimandi, G., Moreau, E. 2017. In vitro cytotoxic effects of DEHP-alternative plasticizers and their primary metabolites on a L929 cell line. Chemosphere 173, 452-459.

Engel, A., Buhrke, T., Imber, F., Jessel, S., Seidel, A., Völkel, W., Lampen, A. 2017. Agonistic and antagonistic effects of phthalates and their urinary metabolites on the steroid hormone receptors ERa, ERß, and AR. Toxicol. Lett. 277, 54-63.

Engel, A., Buhrke, T., Kasper, S., Behr, A. C., Braeuning, A., Jessel, S., Seidel, A., Völkel, W., Lampen, A. 2018. The urinary metabolites of $\mathrm{DINCH}{ }^{\circledR}$ have an impact on the activities of the human nuclear receptors ERa, ER $\beta$, AR, PPARa and PPARY. Toxicol. Lett., 287, 83-91.

ECHA. 2013. Evaluation of new scientific evidence concerning DINP and DIDP in relation to entry 52 of Annex XVII to REACH Regulation (EC) No 1907/2006. https://echa.europa.eu/documents/10162/31b4067e-de40-4044-93e8-9c9ff1960715. Please 
note: DINP is restricted according Annex XVII of Regulation (EC) No 1907/2006 and can therefore not be used for toys which can be put into the mouth by children. https://echa.europa.eu/documents/10162/57096439-2ddd-4f14-b832-85181a09f595

Frederiksen, H., Kuiri-Hänninen, T., Main, K. M., Dunkel, L., Sankilampi, U. 2014. A longitudinal study of urinary phthalate excretion in 58 full-term and 67 preterm infants from birth through 14 months. Environ Health Perspect. 122, 9,998-1005.

Fromme, H., Schütze, A., Lahrz, T., Kraft, M., Fembacher, L., Siewering, S., Burkardt, R., Dietrich, S., Koch, H. M., Völkel, W. 2016. Non-phthalate plasticizers in German daycare centers and human biomonitoring of DINCH metabolites in children attending the centers (LUPE 3). Int. J. Hyg. Environ. Health. 219, 1, 33-39.

Giovanoulis, G., Alves, A., Papadopoulou, E., Cousins, A. P., Schütze, A., Koch, H. M., Haug, L. S., Covaci, A., Magnér, J., Voorspoels, S. 2016. evaluation of exposure to phthalate esters and DINCH in urine and nails from a Norwegian study population. Environ. Res. 151, 80-90.

Giovanoulis, G., Bui, T., Xu, F., Papadopoulou, E., Padilla-Sanchez, J. A., Covaci, A., Haug, L. S., Cousins, A. P., Magnér, J., Cousins, I. T., de Wit, C. A. 2018. Multi-pathway human exposure assessment of phthalate esters and DINCH. Environ. Int. 112, 115-126.

Hauser, R., Calafat, A. M. 2005. Phthalates and human health. Occup. Environ. Med. 62, 11, 806-818.

Huang, L. P., Lee, C. C., Fan, J. P., Kuo, P.H., Shih, T.S., Hsu, P.C. 2014. Urinary metabolites of di(2-ethylhexyl) phthalate relation to sperm motility, reactive oxygen species generation, and apoptosis in polyvinyl chloride workers. Int. Arch. Occup. Environ. Health. $87,6,635-646$.

Huygh, J., Clotman, K., Malarvannan, G., Covaci, A., Schepens, T., Verbrugghe, W., Dirinck, E., Van Gaal, L., Jorens, P. G. 2015. Considerable exposure to the endocrine disrupting chemicals phthalates and bisphenol-A in intensive care unit (ICU) patients. Environ Int. 81, 64-72.

Jeon, S., Kim, K. T., Choi, K. Migration of DEHP and DINP into dust from PVC flooring products at different surface temperature. Sci. Total Environ. 15, 547, 441-446.

Johns LE, Cooper GS, Galizia A, Meeker JD. 2015. Exposure assessment issues in epidemiology studies of phthalates. Environ. Int. 85, 27-39.

Kleeff, J., Kornmann, M., Sawhney, H., Korc, M. 2000. Actinomycin D induces apoptosis and inhibits growth of pancreatic cancer cells. Int. J. Cancer. 86, 3,399-407.

Koch, H. M., Müller, J., Angerer, J. 2007a. Determination of secondary, oxidised di-isononylphthalate (DINP) metabolites in human urine representative for the exposure to commercial DINP plasticizers. J. Chromatogr. B Analyt. Technol. Biomed Life Sci. 847, 2, 114-125.

Koch, H. M., Angerer, J. 2007b. Di-iso-nonylphthalate (DINP) metabolites in human urine after a single oral dose of deuterium-labelled DINP. Int. J. Hyg. Environ. Health. 210, 1, 9-19. 
Koch, H. M., Schütze, A., Pälmke, C., Angerer, J., Brüning, T. 2013. Metabolism of the plasticizer and phthalate substitute diisononyl-cyclohexane-1,2-dicarboxylate (DINCH(®)) in humans after single oral doses. Arch. Toxicol. 87, 5, 799-806.

Koch HM, Rüther M, Schütze A, Conrad A, Pälmke C, Apel P, Brüning T, Kolossa-Gehring M. Phthalate metabolites in 24-h urine samples of the German Environmental Specimen Bank (ESB) from 1988 to 2015 and a comparison with US NHANES data from 1999 to 2012. 2017. Int. J. Hyg. Environ. Health. 220(2 Pt A),130-141.

Lagerberg, J. W., Gouwerok, E., Vlaar, R., Go M., de Korte D. 2015. In vitro evaluation of the quality of blood products collected and stored in systems completely free of di(2ethylhexyl)phthalate-plasticized materials. Transfusion. 55, 3, 522-531.

Nuti, F., Hildenbrand, S., Chelli, M., Wodarz, R., Papini, A. M. 2005. Synthesis of DEHP metabolites as biomarkers for GC-MS evaluation of phthalates as endocrine disrupters. Bioorg. Med. Chem. 13, 10, 3461-3465.

Regulation (EC) No 1272/2008 of the european parliament and of the council. 2008. Official Journal of the European Union.

Regulation (EU) 2017/745 of the European Parliament and of the Council of 5 April 2017 on medical devices. Official Journal of the European Union.

SCENIHR, (Scientific Committee on Emerging and Newly-Identified Health Risks) opinion on the safety of medical devices containing dehp- plasticized pvc or other plasticizers on Neonates and other groups possibly at risk (February 2008). https://ec.europa.eu/health/archive/ph risk/committees/04 scenihr/docs/scenihr o 014.pdf

SCENIHR, (Scientific Committee on Emerging and Newly-Identified Health Risks) opinion on the safety of medical devices containing dehp- plasticized pvc or other plasticizers on Neonates and other groups possibly at risk (February 2016 update). https://ec.europa.eu/health/sites/health/files/scientific committees/emerging/docs/scenihr o 047. pdf

Schütze, A., Pälmke, C., Angerer, J., Weiss, T., Brüning, T., Koch, H. M. 2012. Quantification of biomarkers of environmental exposure to di(isononyl)cyclohexane-1,2-dicarboxylate (DINCH) in urine via HPLC-MS/MS. J. Chromatogr. B. , 895-896, 123-130.

Schütze, A., Kolossa-Gehring, M., Apel, P., Brüning, T., Koch, H. M. 2014. Entering markets and bodies: increasing levels of the novel plasticizer Hexamoll® DINCH® in $24 \mathrm{~h}$ urine samples from the German Environmental Specimen Bank. Int J Hyg Environ Health. 217, 23, 421-426.

Schütze, A., Otter, R., Modick, H., Langsch, A., Brüning, T., Koch, H. M. 2017. Additional oxidized and alkyl chain breakdown metabolites of the plasticizer DINCH in urine after oral dosage to human volunteers. Arch. Toxicol. 91, 1, 179-188.

Silva, M. J., Samandar, E., Preau, J. L. Jr, Reidy, J. A., Needham, L. L., Calafat, A. M. 2007a. Quantification of 22 phthalate metabolites in human urine. J. Chromatogr. B Analyt. Technol. Biomed. Life Sci. 860, 1, 106-112. 
Silva, M. J., Reidy, J. A., Kato, K., Preau, J. L. Jr, Needham, L. L., Calafat, A. M. 2007b. Assessment of human exposure to di-isodecyl phthalate using oxidative metabolites as biomarkers. Biomarkers. 12, 2, 133-144.

Silva, M.J., Jia, T., Samandar, E., Preau Jr, J. L., Calafat, A. M. 2013. Environmental exposure to the plasticizer 1,2-cyclohexane dicarboxylic acid, diisononyl ester (DINCH) in U.S. adults (2000-2012). Environ. Res., 126, 159-163.

Strømmen, K., Lyche, J. L., Blakstad, E. W., Moltu, S. J., Veierød, M. B., Almaas, A. N., Sakhi, A. K., Thomsen, C., Nakstad, B., Brække, K., Rønnestad, A. E., Drevon, C. A., Iversen, P. O. 2016. Increased levels of phthalates in very low birth weight infants with septicemia and bronchopulmonary dysplasia. Environ Int. 89-90, 228-234.

Wittassek, M., Wiesmüller, G. A., Koch, H. M., Eckard, R., Dobler, L., Müller, J. Angerer, J., Schlüter, C. 2007. Internal phthalate exposure over the last two decades--a retrospective human biomonitoring study. Int. J. Hyg. Environ. Health. 210, 3-4, 319-333.

Ye, H., Ha, M., Yang, M., Yue, P., Xie, Z., Liu, C. 2017. Di2-ethylhexyl phthalate disrupts thyroid hormone homeostasis through activating the Ras/Akt/TRHr pathway and inducing hepatic enzymes. Sci. Rep. 7, 40153.

Zeman, F. A., Boudet, C., Tack, K., Floch-Barneaud, A., Brochot, C., Péry, A. R., Oleko, A., Vandentorren, S. 2013. Exposure assessment of phthalates in French pregnant women: results of the ELFE pilot study. Int. J. Hyg. Environ. Health. 216, 3, 271-279.

Zhang, S., Ma J., Fu, Z., Zhang, Z., Cao, J., Huang, L., Li, W., Xu, P., Cao, X. 2016. Promotion of breast cancer cells MDA-MB-231 invasion by di(2-ethylhexyl)phthalate through matrix metalloproteinase-2/-9 overexpression. Environ. Sci. Pollut. Res. Int. 23, 10, 97429749. 
Highlights

- 7-oxo-MMeOCH and 5-oxo-MEHP induced no action on cell proliferation at $0.1 \mathrm{mg} / \mathrm{mL}$

- Among secondary metabolites of DEHP, 5-oxo-MEHP induced an action on L929

- Among secondary metabolites of $\mathbf{M M e O C H}, 7-\mathbf{0 x o - M M e O C H}$ induced an action on $\mathbf{L 9 2 9}$

- Secondary metabolites of $\mathbf{M M e O C H}$ are less toxic than $\mathbf{M M e O C H}$

- Secondary metabolites of MMeOP are not toxic like $\mathbf{M M e O C H}$ 


\section{Graphical abstract}

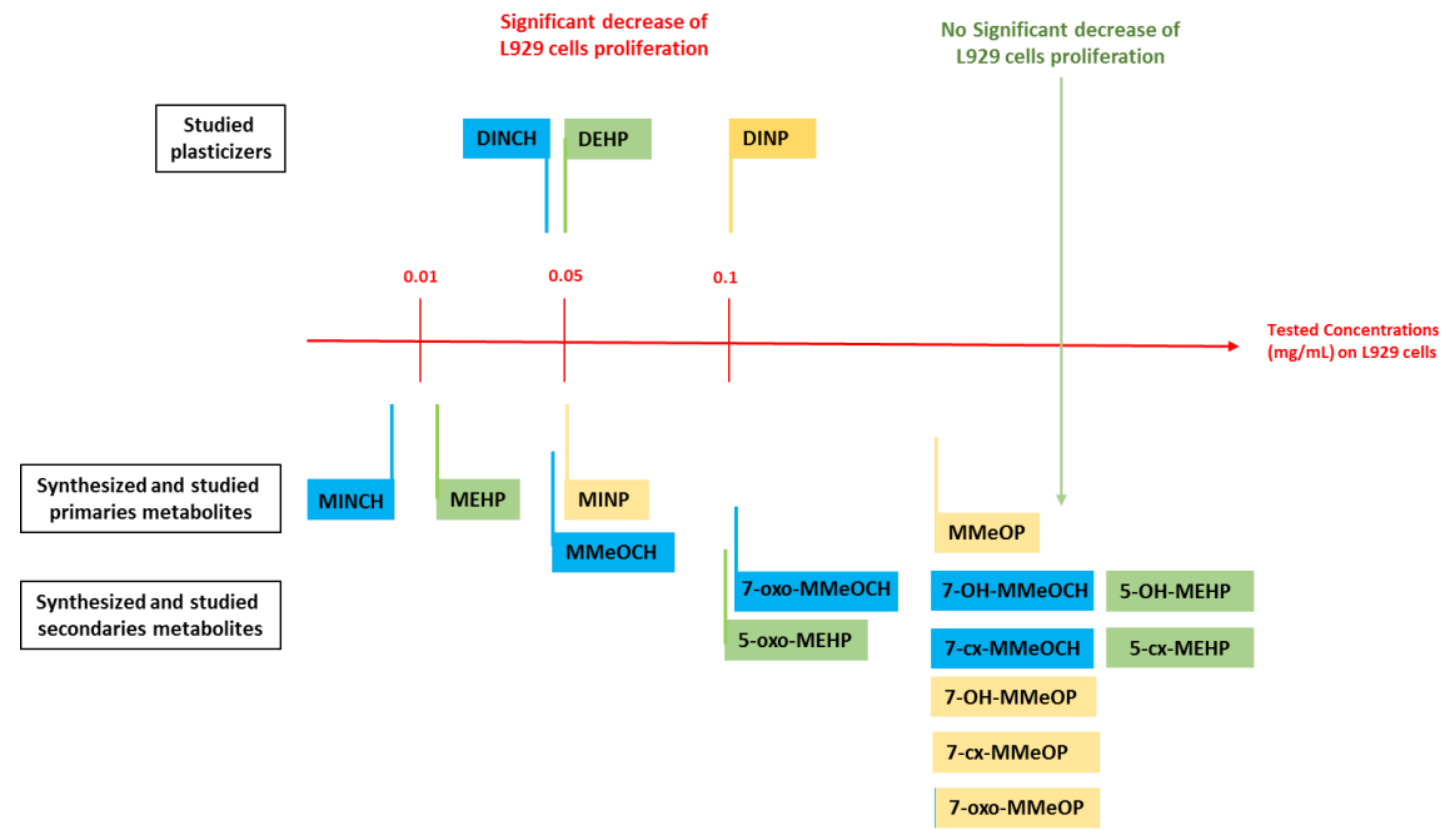

